\title{
Electrophoretic Properties of Latex Particles with Immobilized Bovine Serum Albumin
}

\author{
Tai-Horng Young, ${ }^{*}, 1$ Chen-Yuan Kao, $†$ Meng-Ying Hsieh, $\uparrow$ Bing-Jie Wang, $\nmid$ and Jyh-Ping Hsu $\dagger$ \\ *Institute of Biomedical Engineering, College of Medicine and College of Engineering, National Taiwan University, Taipei, Taiwan 10016, \\ Republic of China; and $\dagger$ Department of Chemical Engineering, National Taiwan University, Taipei, Taiwan, 10617, Republic of China
}

Received November 20, 2000; accepted March 22, 2001

\begin{abstract}
In this study, bovine serum al bumin (BSA) was covalently immobilized onto the surfaces of methyl methacrylate/methyl acrylic acid core-shell latex particles by the carbodiimide method, and the variation of thezeta potential of theBSA-latex complex as a function of the amount of BSA immobilized was measured. The experimental data revealed that the absolute zeta potential of BSA- latex complex exhibited a local minimum at $0.05 \mathrm{mg} / \mathrm{m}^{2}$ of immobilized BSA. A two-layer model for the description of the electrophoretic behavior of the BSA-latex complex is proposed which is capable of explaining the behavior of the variation of the zeta potential as a function of the amount of BSA immobilized. (2) 2001 Academic Press

Key Words: zeta potential; BSA-latex; particles; a two-layer model.
\end{abstract}

\section{INTRODUCTION}

Protein-coated colloid particles are used as a convenient and versatile tool in the field of biotechnology and medicine for various immunoassays and diagnostic tests based on the antigenantibody reaction (1). The particles that can be used to attach either an antigen or the corresponding antibody include erythrocytes, bacteria, gelatin particles, liposomes, metal sols, and various kinds of polymer latex particles. Recent advances in polymer science have made it possible to produce uniform polymer latex particles with the desired functional groups which can be used for the covalent immobilization of proteins. Rembaum et al. (2) copolymerized methacrylic acid and 2-hydroxyl methacrylate latex particles by means of ionizing radiation. Marumoto et al. (3) prepared polymer latex particles by emulsion copolymerization of methyl methacrylate, methacrylic acid, 2-hydroxyl methacrylate, and ethylene glycol dimethacrylate, using sodium dodecylbenzene sulphonate as an emulsifier. Kondo et al. (4) prepared latex particles having carboxylated on their surfaces by copolymerization of styrene and acrylamide by emulsifierfree emulsion polymerization. More recently, the core-shell type polymer particles coated by proteins were used in immunochemical studies as was reported by Quesada et al. (5). Overall, these

\footnotetext{
${ }^{1}$ To whom correspondence should be addressed. E-mail: thyoung@ha.mc. ntu.edu.tw.
}

polymer latex surfaces contain functional groups to which the proteins can be covalently coupled.

In this paper, latex particles composed of a poly (methyl methacrylate) (PMMA) core and a poly (methyl methacrylateco-methacrylic acid) (PMMA-co-PMAA) shell were synthesized. Bovine serum albumin (BSA) was covalently bonded onto the surface of the latex particles to form the immunolatices. The main purpose of our investigation is to achieve better insight into the electrophoretic properties of protein-coated colloid particles. Many academic studies have been performed to understand the complex phenomena of microscopic polymer latex particles as a function of ionic strength, $\mathrm{pH}$, and temperature. By electrophoretic mobility measurements of latex particles, the surface structures of the latex particles can be analyzed with a suitable model. Nakamura et al. (6) measured the electrophoretic mobilities of antigen and antibody-carrying latex particles at different $\mathrm{pH}$ and ionic strength. Carboxylated latex particles were used and proteins were fixed on the latex particle surface through chemical bonding. The sizes of the proteins were estimated by the approximate formula that directly relates the mobility to the charge density in the surface layer. Folkersma et al. (7) reported the zeta potential of polystyrene spheres as a function of ionic strength shows a maximum value, which is different from the expected decrease in latex mobility with increasing electrolyte concentration. A model consisting of surface conductance and hairy layers was used by Folkersma et al. to explain the electrophoretic properties of polystyrene spheres. Nabzar et al. (8) investigated the electrokinetic properties and colloidal stability behavior of core-shell latexes bearing a thermosensitive shell. It was pointed out that the electrophoretic mobility and particle size are temperature-sensitive. Casals et al. (9) monitored the distribution of charged phospholipids in the membrane of liposomes, which bear different properties of either positive or negative charge. An extensive study was performed by Makino et al. (10) on the distribution of charges in the microcapsule surfaces to know the effects of surface composition upon surface structure. Therefore, the zeta potential measurements are useful to know the effects of immobilized BSA concentration upon the electrophoretic properties of our prepared latex particles. Unexpectedly, the zeta potential of the prepared BSA-coated latices exhibits a local minimum as the concentrations of immobilized 
BSA increases. Apparently, the structure of the attached BSA layer on a latex particle plays a significant role. In an attempt to elaborate the phenomenon observed, a model for proteins, which composes two BSA sublayers with different structures and properties, is proposed.

\section{EXPERIMENTAL}

\section{Materials}

BSA (fraction V, and containing more than $99 \%$ monomeric albumin) was obtained from Sigma. All other chemicals used were of reagent grade. Methyl methacrylate (MMA) and methyl acrylic acid (MAA) were distilled under nitrogen atmosphere and reduced pressure prior to polymerization. Other materials were used as received without further purification. Water was double distilled and deionized before use.

\section{Preparation of Latexes}

The core-shell type polymer latex was synthesized by a twostep emulsifier-free emulsion polymerization process. Potassium persulfate was used as initiator. In the first step, the core of the polymer latex was prepared at $80^{\circ} \mathrm{C}$ under nitrogen atmosphere at a stirring speed of $300 \mathrm{rpm}$ by batch emulsion polymerization of MMA. After $1 \mathrm{~h}$ of polymerization, the core of the polymer latex was quenched to room temperature. In the second stage, the emulsion copolymerization of MMA and MAA was carried out to put the shell onto the core synthesized in the first step. At first, water and monomers were added to the reactor to swell the seed particles for $24 \mathrm{~h}$ at room temperature. The molar fraction of copolymerized MAA was 0.3 . The initiator was then added to the swelled system to begin the copolymerization. The reaction was allowed to proceed for $1 \mathrm{~h}$ at $70^{\circ} \mathrm{C}$ under continuous stirring at $300 \mathrm{rpm}$ and nitrogen gas was continuously passed through the reactor.

These latices were purified by dialysis using Spectra/Pro molecularporous membrane tubeing (MWCO: 8000, Spectrum Laboratories, Inc., USA and Canada) for 1 week. The average size of particles was estimated by measuring the diameter of about 10 particles in several scanning electron microscopic photographs and was determined to be $330 \pm 5 \mathrm{~nm}$. The surface area of the latex particle was calculated from the average diameter. The latex concentrations were determined by the dry weight method.

\section{Covalent Immobilization of Proteins onto Latex Particles}

BSA was covalently immobilized onto the latex particles by the carbodiimide method. BSA was dissolved in phosphate buffer at pH 5 and ionic strength 0.002 . The BSA solution of desired concentrations $(10 \mathrm{ml})$ were mixed with $10 \mathrm{ml}$ of the latex suspensions $\left(15 \mathrm{mg} / \mathrm{cm}^{3}\right)$. BSA was adsorbed onto the latex particles by equilibrating in a shaker bath at room temperature for $2 \mathrm{~h}$. Subsequently, BSA was covalently coupled by addition of $10 \mathrm{mg}$ of 1-Ethyl-3-(3-Dimethylamine propyl) carbodiimide dissolved in water for $4 \mathrm{~h}$. After the coupling reaction, the reaction mixture was centrifuged for $30 \mathrm{~min}$ at $12000 \mathrm{rpm}$ and then the absorbance of the supernatant fluid at $280 \mathrm{~nm}$ was checked to determine the amount of BSA bound to the latex surface by the difference between the initial and the supernatant protein concentrations. Every coupling reaction was repeated four times. No protein was detected in the supernatant when the initial amount of BSA added to the latex suspensions was less than $0.7 \mathrm{mg}$. Hence, it was assumed that all BSA were bound to the latex surface in this circumstance with low BSA concentration in the solution. However, the standard deviation of the BSA concentration in the supernatant was less than $5 \%$ of the average value at higher BSA concentration in the solution.

\section{Zeta Potential Measurements}

Electrophoretic measurements of latex particles as a function of the amount of bound BSA were performed at $25^{\circ} \mathrm{C}$ in a Zetasizer 2000 (Malvern Instruments, U.K.) based on the laser-Doppler microelectrophoresis. The sediments of BSAimmobilized particles were resuspended and diluted to a concentration of $150 \mu \mathrm{g} / \mathrm{ml}$ with phosphate buffer at $\mathrm{pH} 7.4$ and ionic strength of 0.09 . The results of zeta potential measurements are the average of three measurements at the stationary level.

\section{THEORY}

Intuitively, since the attachment of BSA to the latex particle is of covalent nature, monolayer coverage is expected. However, the free end of an attached BSA is also capable of joining with another BSA by carbodiimide. Therefore, we assume that the BSA-latex complex can be simulated by a rigid core covered by a porous membrane layer. For simplicity, the two-layer model shown in Fig. 1 is considered. If the amount of BSA attached to the surface of a latex particle is smaller than that corresponding to a monolayer coverage, a mono-layer membrane is considered. If the amount of BSA attached to particle surface exceeds that, then a two-layer membrane is assumed. In our case, the size of a latex particle $(\cong 330 \mathrm{~nm})$ is much larger than that of BSA

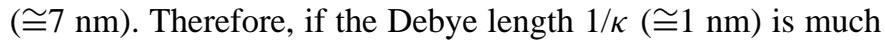
smaller than the size of a latex particle, then the surface of the particle can be regarded as a planar surface. In this case only a one-dimensional problem needs to be solved. Let us consider the approximate model shown in Fig. 2, where $d$ denotes the thickness of a BSA layer. Suppose that the latex particle is rigid and the BSA layers are ion-penetrable. The particle moves with velocity $U$ in an electrolyte solution as a response to an applied electric field $E$, which is parallel to surface of the particle. The $x$ axis is perpendicular to the surface of the particle and the origin is located at the particle-BSA interface. Suppose that the spatial variations in the electric potential and the flow field can be described respectively by the Poisson equation and the NavierStokes equation $(11,12)$. We have, for the mono-layer case,

$$
\varepsilon \frac{d^{2} \Phi}{d x^{2}}=-\rho_{\mathrm{el}}-j z_{1} F C_{1}
$$




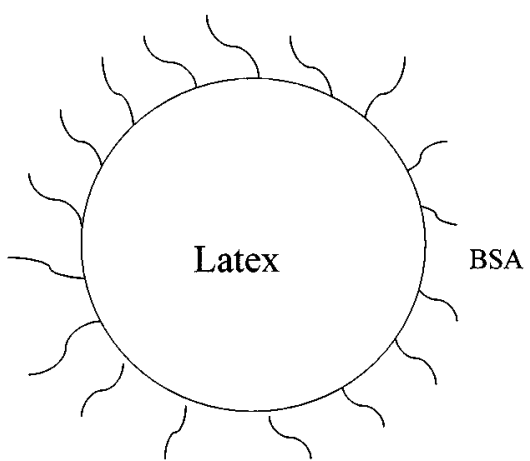

(a)

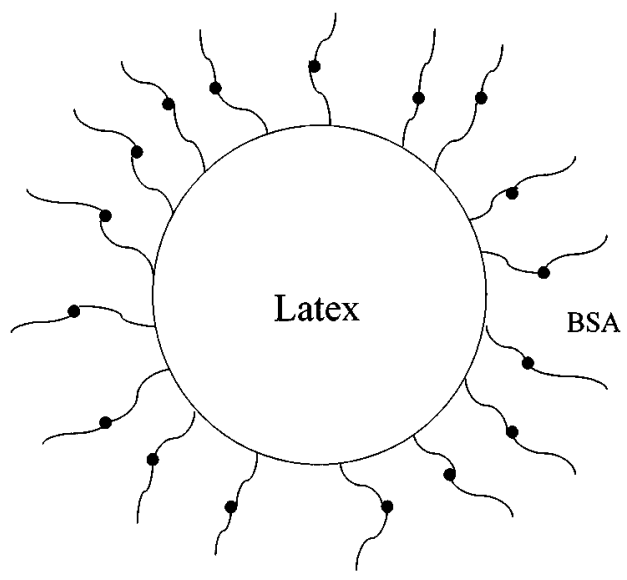

(b)

FIG . 1. Schematic representation of the attachment of BSA molecules onto the surface of a latex particle: (a) mono-layer model, (b) two-layer model.

$$
\eta \frac{d^{2} u}{d x^{2}}=-\rho_{\mathrm{el}} E+j \gamma_{1} u
$$

In these expressions, $j=1$ for $0<x<d$, and $j=0$ for $d<x$. $\Phi$ is the electric potential, $u$ is the fluid velocity, $\varepsilon$ and $\eta$ are, respectively, the dielectric permittivity and the viscosity, $\rho_{\mathrm{el}}$ is the space charge density, $F$ is the Faraday's constant, and $z_{1}$, $C_{1}$, and $\gamma_{1}$ are the valence of the fixed charge, the concentration of fixed charge, and the friction coefficient of the BSA layer. The boundary conditions associated with Eqs. [1] and [2] are assumed as

$$
\begin{gathered}
\Phi=\Phi_{0} \quad \text { and } \quad u=0 \quad \text { at } x=0 \\
\left.\Phi\right|_{d-} ^{d+}=\left.\frac{d \Phi}{d x}\right|_{d_{-}} ^{d+}=\left.u\right|_{d-} ^{d+}=\left.\frac{d u}{d x}\right|_{d-} ^{d+}=0 \\
\Phi=0 \quad \text { and } \quad u=-U \quad \text { as } x \rightarrow \infty
\end{gathered}
$$

Here $\Phi_{0}$ is the electric potential at the particle-BSA interface. Equation [3] implies that the fluid velocity vanishes on the slipping plane (particle-BSA interface). Equation [4] implies that $\Phi, d \Phi / d x, u$, and $d u / d x$ are all continuous at $d$. Equation [5] states that bulk liquid velocity has the same magnitude as that of the electrophoretic velocity of the particle, but in the opposite direction.

Similarly, for the two-layer case, the spatial variations in the electric potential and the flow field can be described, respectively, by

$$
\varepsilon \frac{d^{2} \Phi}{d x^{2}}=-\rho_{\mathrm{el}}-j z_{2} F C_{2}
$$

and

$$
\eta \frac{d^{2} u}{d x^{2}}=-\rho_{\mathrm{el}} \cdot E+j \gamma_{2} u
$$

where $j=1$ for $0<x<2 d$, and $j=0$ for $2 d<x . z_{2}, C_{2}$, and $\gamma_{2}$ are the valence of the fixed charge, the concentration of fixed charge, and the friction coefficient of the BSA layer. The boundary conditions associated with Eqs. [6] and [7] are assumed as

$$
\begin{gathered}
\Phi=\Phi_{0} \quad \text { and } \quad u=0 \quad \text { at } x=0 \\
\left.\Phi\right|_{2 d-} ^{2 d+}=\left.\frac{d \Phi}{d x}\right|_{2 d-} ^{2 d+}=\left.u\right|_{2 d-} ^{2 d+}=\left.\frac{d u}{d x}\right|_{2 d-} ^{2 d+}=0
\end{gathered}
$$

Latex

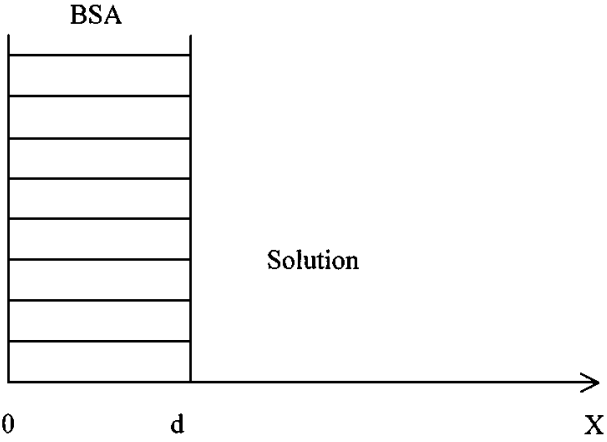

(a)

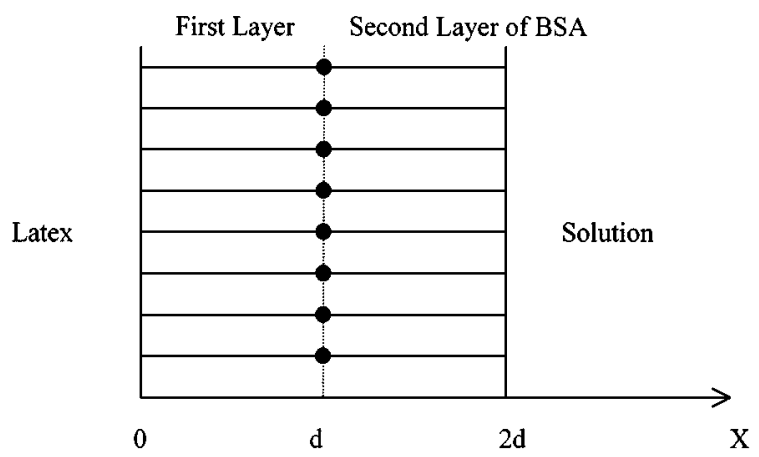

(b)

FIG.2. Schematic representation of the simplified model considered, where $d$ is the thickness of a BSA mono-layer: (a) mono-layer model, (b) two-layer model. 


$$
\Phi=0 \quad \text { and } \quad u=-U \quad \text { as } x \rightarrow \infty
$$

Suppose that the spatial variation in the concentration of ionic species, $N_{i}(x)$, follows the Boltzmann distribution

$$
N_{i}(x)=N_{i}^{0} \exp \left(\frac{-v_{i} e \Phi}{k_{\mathrm{B}} T}\right)
$$

where $e$ is the elementary charge, $k_{\mathrm{B}}$ is the Boltzmann's constant, $T$ is the absolute temperature, and $\nu_{i}$ and $N_{i}^{0}$ are, respectively, the valence and the bulk concentration of ionic species $i$. Intuitively, both the concentration of fixed charge and the friction coefficient are related to the nature of a BSA layer. For simplicity, we assume the following averaged empirical relations:

$$
\begin{aligned}
z_{i} C_{i} & =k_{1} B^{n_{1}}, \quad i=1,2, \\
\gamma_{i} & =k_{2} B^{n_{2}}, \quad i=1,2 .
\end{aligned}
$$

In these expressions, $B$ represents the density of a BSA layer, and $k_{i}$ and $n_{i}, i=1,2$, are constant, which characterize the nature of a BSA layer. The electrophoretic mobility $\mu(=U / E)$ can be estimated by solving numerically Eqs. [1], [2], [6], and [7] simultaneously subject to Eqs. [3]-[5] and [8]-[10], followed by applying the Smoluchowsk's formula for $\mu$ and zeta potential.

\section{RESULTS AND DISCUSSION}

The experimental data for the variation in the zeta potential of BSA-latex complex is illustrated in Fig. 3. The results for small amount of BSA attached to the surface of latex particle (measured by $B$ ) are magnified to show its nature. Figure 3 reveals that, if $B$ is small, the absolute zeta potential increases with $B$. It exhibits a local minimum at $B \cong 0.05 \mathrm{mg} / \mathrm{m}^{2}$, and then approaches roughly to a constant value with a further increase in $B$. Peula et al. (13) have revealed that if the adsorption of protein to latex is of physical nature, the electrophortic mobility of protein-latex complex varies monotonically with the amount of adsorption. Therefore, the existence of the local minimum of zeta potential against the amount of adsorption implies that the adsorption of BSA to latex particle is not pure physical in our case.

The data shown in Fig. 3 suggests that if $B<0.0726 \mathrm{mg} / \mathrm{m}^{2}$, a monolayer model should be used, and if $B>0.0726 \mathrm{mg} / \mathrm{m}^{2}$, a two-layer model should be used. The fitted values based on the present model are also presented in Fig. 3, and the values of the adjustable parameters are summarized in Table 1 . The existence of a local minimum in the zeta potential as $B$ varies can be explained as follows. The absolute zeta potential increases with the amount of charges associated with a latex particle, which increases with the amount of BSA attached onto its surface. On the other hand, the resistance to liquid flow due of the presence of a BSA layer also increases with the amount of BSA attached. If $B$ is small, the former dominates, and the absolute zeta potential increases with $B$; however, if $B$ is large, the latter becomes
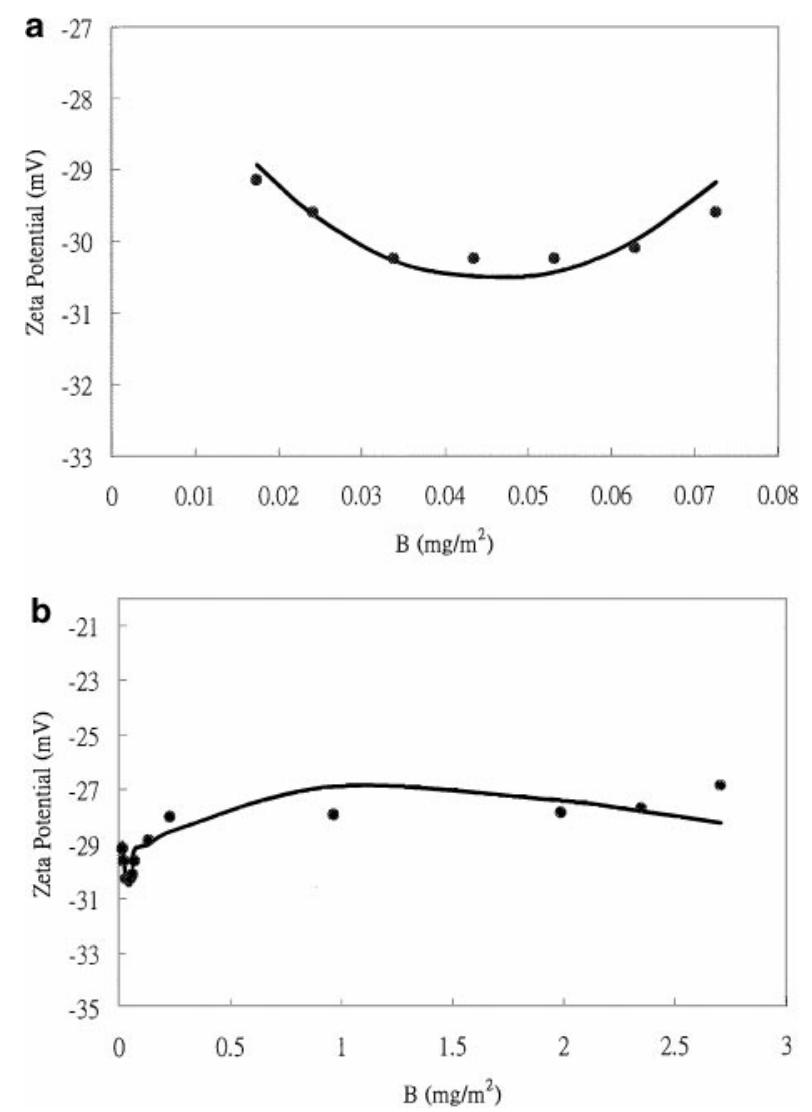

FIG. 3. Variation of the zeta potential of the BSA-latex complex as a function of the surface density of immobilized BSA molecule, $B$. The open circles are the experimental data, and the solid curve denotes the theoretical values based on $\varepsilon=6.95 \times 10^{-10} \mathrm{CV}^{-1} \mathrm{~m}^{-1}, T=298 \mathrm{~K}, \eta=0.79 \times$ $10^{-3} \mathrm{Kg} \mathrm{m}^{-1} \mathrm{~s}^{-1}, v_{+}=-v_{-}=1$, ionic strength $=0.09 \mathrm{M}$, and $d=7 \mathrm{~nm}$. The results for small $B$ are magnified in (a).

more significant, and the absolute zeta potential decreases with the increase in $B$.

Table 1 reveals that the values of $k_{2}$ and $n_{2}$ in the two-layer model are smaller than the corresponding values in the monolayer model. Referring to Fig. 1, the average void fraction of BSA-latex complex for the case of two-layer model is larger than that for the case of mono-layer. This implies that the average resistance to liquid flow provided by the BSA layer for the former is smaller than that for the latter. The negative sign of $k_{1}$ suggests

\section{TABLE 1}

Values of the Parameters U sed in Fig. 3 for the Case $\varepsilon=$ $6.95 \times 10^{-10} \mathrm{CV}^{-1} \mathrm{~m}^{-1}, T=298 \mathrm{~K}, \eta=0.79 \times 10^{-3} \mathrm{Kg} \mathrm{m}^{-1} \mathrm{~s}^{-1}$, $\nu_{+}=-\nu_{-}=1$, ionic strength $=0.09 \mathrm{M}, \Phi_{0}=-27 \mathrm{mV}, d=7 \mathrm{~nm}$

\begin{tabular}{lcccc}
\hline & $k_{1}$ & $k_{2}$ & $n_{1}$ & $n_{2}$ \\
\hline 1st layer & -35 & $9 \times 10^{15}$ & 1 & 2.7 \\
2nd layer & -20 & $9 \times 10^{13}$ & 1 & 1.1 \\
\hline
\end{tabular}

Note. The first and the second layers intersect at $B=0.07257 \mathrm{mg} / \mathrm{m}^{2}$. 
that the BSA layer is negatively charged, which is justified by the sign of zeta potential. The observation that the absolute value of $k_{1}$ for the case of mono-layer model is larger than that for the case of two-layer model can be explained again by the magnitude of the averaged void fraction of a BSA layer.

The linear size of latex particle and that of BSA molecule are 330 and $7 \mathrm{~nm}$, respectively. The average molecular weight of BSA molecule is about $70000 \mathrm{~g} / \mathrm{mol}$, and the molar fraction of copolymerized MAA is 0.3 . Assuming that the BSA molecule is cubic, then the theoretical mono-layer coverage of BSA bound unto the latex surface corresponds to $B \cong 0.712$ $\mathrm{mg} / \mathrm{m}^{2}$, which is larger than that estimated based on the experimental data. This can be explained by the steric hindrance for the attachment of BSA molecule to the carboxyl group on the latex surface.

The two-layer model used in the present study is an idealized model. A more realistic model should assume that the spatial distribution of the density of adsorbed BSA layer is a function of the distance from latex surface. Unfortunately, the experimental data at hand are insufficient for the determination of this function.

\section{ACKNOWLEDGMENT}

This work is supported by the National Science Council of the Republic of China.

\section{REFERENCES}

1. Nakamura, R. M., Kasahara, Y., and Rechnitz, G. A., "Immunochemical Assays and Biosensor Technology for the 1990s," American Society for Microbiology, Washington, DC, 1992.

2. Rembaum, A., Yen, S. P. S., Cheong, E., Wallace, S., Molday, R. S., Cordon, I. L., and Dreyer, W. J., Macromolecules 9, 328 (1976).

3. Marumoto, K., Suzuta, T., Noguchi, H., and Uchida Y., Polymer 19, 867 (1978).

4. Kondo, A., Yamasaki, R., and Higashitani, K., J. Ferment. Bioeng. 74, 226 (1992).

5. Quesada, M., Puig, J., Delgado, J. M., Peula, J. M., Molina, J. A., and Hidalgo-Alvarez R., Colloids Surf. B 8, 303 (1997).

6. Nakamura, M., Ohshima, H., and Kondo, T., J. Colloid Interface Sci. 149, 241 (1992).

7. Folkersma, R., van Diemen, A. J. G., and Stein H. N., Langmuir 14, 5973 (1998).

8. Nabzar, L., Duracher, D., Elaissari, A., Chauveteau, G., and Pichot, C., Langmuir 14, 5062 (1998).

9. Casals, E., Soler, M., Gallardo, M., and Estelrich, J., Langmuir 14, 7522 (1998).

10. Makino, K., Umetsu, M., Goto, Y., Kikuchi, A., Sakurai, Y., Okano, T., and Ohshima H., J. Colloid Interface Sci. 218, 275 (1999).

11. Bird, R. B., Stewart, W. E., and Lightfoot E. N., "Transport Phenomena," Wiley, New York, 1960.

12. Ohshima, H., Nakamura, M., and Kondo, T., Colloid Polym. Sci. 270, 873 (1992).

13. Peula, J. M., Callejas, J., and de las Nieves, F. J., in "Surface Properties of Biomaterials" (R. West and G. Batts, Eds.), Butterworth-Heinemann, London, 1994. 